\title{
NOTA TÉCNICA
}

\section{RENDIMIENTO DE NUEVOS HÍBRIDOS PUMAS DE MAÍZ PARA LA ZONA DE TRANSICIÓN DE MÉXICO ${ }^{1}$}

\author{
Rafael Martínez $z^{2}$,Margarita Tadeo², Angel Piña Del Valle², Alejandro Espinosa ${ }^{3}$, Ana María Solano ${ }^{2}$
}

\begin{abstract}
RESUMEN
Rendimiento de nuevos híbridos PUMAS de maíz para la zona de transición de México. Con el objetivo de ofrecer nuevas y mejores opciones a los productores de la Zona de Transición Bajío Valles Altos (1800-2200), se evaluaron cuatro nuevos híbridos de cruza doble y 16 de cruza triple y se compararon con híbridos comerciales H-135, A791, PUMA 1157 y PUMA 1159, trilineales y de cruza doble el H-137, en el ciclo primavera-verano de 1996. El análisis de varianza mostró diferencias estadísticas altamente significativas, para casi todas las variables evaluadas en cuanto al factor genotipos y no significativas para el factor repeticiones. Nueve híbridos trilineales mostraron rendimientos de ocho a 12 t/ha, superando estadísticamente al resto de los híbridos tanto dobles como trilineales, incluyendo los testigos. Destacó el híbrido trilineal UHT96E410 con 12,4 t/ha y con 77 días a floración masculina, siendo el testigo A-791, también trilineal, el que más se les acercó con 8,6 toneladas y con 94 días a la floración masculina, además de que UHT96E410 tuvo $209 \mathrm{~cm}$ de altura. Cinco de los testigos tuvieron alturas de planta de más de $230 \mathrm{~cm}$. Así es como sobresalen estos nuevos híbridos con excelentes rendimientos y características agronómicas favorables, ampliando a su vez el rango de opciones para los agricultores de la zona de transición de México.
\end{abstract}

\begin{abstract}
Yield of new PUMAS (Zea mays L.) corn hybrids for transitional zones in Mexico. Four new double-cross hybrids and 16 triple-cross hybrids were evaluated and compared with triple-cross commercial hybrids H-135, A791, PUMA 1157 and PUMA 1159, and double-cross H-137, during the 1996 spring-summer season. The purpose was to offer new and better options to producers in the Bajio and Highland Transitional Zones in Mexico (1800-2200 m.a.s.1.). Analysis of variance showed highly significant statistical differences for almost all variables for which the genotype factor was studied, and non-significant differences for repetitions. Nine triple-cross hybrids yielded between 8 and $12 \mathrm{t} / \mathrm{ha}$, a greater yield than that of the rest of the hybrids (double and triple crosses), and also greater than that of the controls. The triple-cross UHT96E410 hybrid stood out yielding 12,4 t/ha in 77 days between sowing and tasseling. The triple-cross control A-791 followed, yielding 8.6 t/ha in 99 days between sowing and tasseling; UHT96E410 grew up to $209 \mathrm{~cm}$ in height. Five of the controls showed heights of over $230 \mathrm{~cm}$. These new hybrids stood out for their superior yield and favorable agronomic traits, offering farmers in the transitional zones of Mexico a broader variety of options.
\end{abstract}

\section{INTRODUCCIÓN}

La zona de transición entre el Bajío-Valles Altos (1800-2200 msnm) de México es una área geográfica que posee condiciones agroclimáticas de alto potencial de producción para el cultivo de maíz. Así mismo, el uso de semilla mejorada es elevado, rebasando el $70 \%$ de la superficie que se dedica a esta gramínea (Tadeo et $a l, 1996)$. Ello es debido a que en los últimos años se ha incrementado la participación de las empresas semilleras ofreciendo híbridos que son mejores a los comerciales actualmente en uso, como es el H-135 (Cruz et

\footnotetext{
1 Presentado en la XLIII Reunión Anual del PCCMCA. Panamá, 1997.

2 Cátedra de Producción y Tecnología de semilla. FESC-UNAM, Km. 2,5 Carretera Cuautitlán-Teoloyucan, C.P. 54700, Apartado Postal 25, Cuautitlán, Edo. México, México. E-mail: marga@mpsnet.com.mx.

3 Líder Nacional de Semillas, SAGAR, INIFAP, Serapio Rendón 83, México, D.F. E-mail: espinoal@inifap.conacyt.mx
} 
al, 1995; Tadeo 1994). Esto aunado a la disposición de los productores al aceptar las nuevas tecnologías, dado que, si la agricultura es su "modus vivendi" tienen que ir buscando aquellas semillas que les sean más redituables. Tratando de aprovechar esto y teniendo como antecedente los híbridos PUMA 1159 y PUMA 1157 que a inicios de la década de los 90's destacaron en las evaluaciones realizadas en diferentes localidades (Cruz et al, 1995; Espinosa et al, 1995a; Espinosa et al, 1995b), en la UNAM se han venido generando una serie de híbridos que a través de diversas evaluaciones en varias localidades han sobresalido (Tadeo et al, 1996), una de las cuales es tema del presente trabajo, han demostrado que tienen tan buenas características como los dos anteriores, lo cual les la oportunidad de ser utilizados por los productores de la zona de transición entre el BajioValles Altos de la República Mexicana.

Se estableció como objetivo el evaluar el rendimiento de los nuevos híbridos de la UNAM en comparación con los testigos comerciales de la zona de transición el Bajío-Valles Altos, así como el definir las perspectivas de su utilización por parte de los productores de esta región del país.

\section{MATERIALES Y MÉTODOS}

El experimento se estableció en el ciclo primavera-verano de 1996 bajo condiciones de punta de riego en las parcelas experimentales de la UNAM Campus Cuautitlán.

Se evaluaron cuatro nuevos híbridos de cruza doble y 16 de cruza triple y se compararon con los híbridos comerciales H-135, A-791, PUMA 1157 y PUMA 1159, triliniales, y de cruza doble el H-137.

La parcela experimental tuvo un surco de $3 \mathrm{~m}$ de largo espaciado a X $0,82 \mathrm{~m}$. La densidad de población obtenida fue de 65000 plantas/ha.

Se utilizó un diseño experimental de bloques completos al azar con tres repeticiones, un análisis de varianza y una comparación de medias Tukey al 0,05 de probabilidad.

Las variables evaluadas fueron: rendimiento, floración y altura de planta y mazorca, mazorcas buenas y malas, plantas acamadas, plantas cosechada, cuateo, calificación y cobertura de mazorca.
Cuadro 1. Tipo de cruza para los diferentes híbridos de maíces simples y dobles de la UNAM en Cuautitlán, México, 1996.

\begin{tabular}{lll}
\hline Híbrido & Origen & Tipo de híbrido \\
\hline UHT96E401 & $5 \times 14$ & Cruza triple \\
UHT96E402 & $6 \times 4$ & Cruza triple \\
UHT96E403 & $7 \times 41$ & Cruza triple \\
UHT96E404 & $7 \times 14$ & Cruza triple \\
UHT96E405 & BHEP $\times 666$ & Cruza triple \\
UHT96E406 & BHEP $\times 673$ & Cruza triple \\
UHT96E407 & $207 \times$ BHEP & Cruza triple \\
UHT96E408 & CS3 $\times 680$ & Cruza triple \\
UHT96E409 & CS2 $\times 680$ & Cruza triple \\
UHT96E410 & CS1 $\times 680$ & Cruza triple \\
UHT96E411 & CS2 $\times 667$ & Cruza triple \\
UHT96E412 & CS3 $\times 671$ & Cruza triple \\
UHT96E413 & CS1 $\times 667$ & Cruza triple \\
UHT96E414 & CS1 $\times 664$ & Cruza triple \\
UHT96E415 & CS1 $\times 58$ & Cruza triple \\
UHT96E416 & CS3 $\times 58$ & Cruza triple \\
UHT96E417 & CS3 $\times 57$ & Cruza triple \\
UHT96E418 & CS2 $\times 58$ & Cruza triple \\
UHT96E419 & CS1 $\times 671$ & Cruza triple \\
UHT96E420 & CS3 $\times 667$ & Cruza triple \\
PUMA 1075T & & Cruza triple \\
H-135 & & Cruza triple \\
A-791 & & Cruza triple \\
H-137 & & Cruza triple \\
P-1157 & & Cruza triple \\
P-1159 & & Cruza triple \\
\hline
\end{tabular}

\section{RESULTADOS Y DISCUSIÓN}

En el Cuadro 2 se observa el análisis de varianza, el cual mostró diferencias estadísticas altamente significativas para casi todas las variables evaluadas en cuanto al factor tratamientos lo cual indica que los genotipos evaluados son diferentes entre si.

En el Cuadro 3 se incluye la comparación de medias y se aprecia que nueve de los nuevos híbridos de la UNAM tienen los más altos rendimientos, que van de ocho a $12 \mathrm{t} / \mathrm{ha}$, y es que por ejemplo el UHT96E410 y el UHYT96E415, tienen la misma hembra en su conformación, razón por la cual tienen similar rendimiento, además de que fue el más alto, similar sucedió con el UHT96E416 y UHT96E418. El testigo que más se les acercó, que es estadística pero no numéricamente igual a ellos, es el A-791 con 8,6 t/ha y en seguida se observan más híbridos trilineales, incluyendo al P-1157 con ocho t/ha, seguidos de los híbridos de cruza doble, 
Cuadro 2. Cuadrados medios y significancia estadística de variables evaluadas en híbridos de maíces simples y dobles de la UNAM en Cuautitlán, México, 1996.

\begin{tabular}{lccrc}
\hline Variable & Tratamientos & Repeticiones & Medias & C.V. \% \\
\hline Floración Masculina & $75,13 * *$ & $7,70 \mathrm{NS}$ & 82,9 & 2,5 \\
Floración Femenina & $85,94 * *$ & $8,80 \mathrm{NS}$ & 84,5 & 2,7 \\
Altura de planta & $1047,50 * *$ & $266,60 \mathrm{NS}$ & 218,8 & 6,0 \\
Altura de mazorca & $860,60 * *$ & $58,82 \mathrm{NS}$ & 136,7 & 6,7 \\
Plantas acamadas & $0,61 * *$ & $0,243 \mathrm{NS}$ & 1,2 & 25,1 \\
Sanidad de mazorca & $1,30 * *$ & $0,000 \mathrm{NS}$ & 8,2 & 6,7 \\
Rendimiento & $9.912,253 * *$ & $872,936 \mathrm{NS}$ & $8.356,5$ & 14,9 \\
\hline
\end{tabular}

Cuadro 3. Comparación de medias (Tukey 0,05) de rendimiento, floración masculina, altura de planta y mazorca y sanidad de mazorca de híbridos de maíz PUMAS evaluados en la UNAM en 1996.

\begin{tabular}{|c|c|c|c|c|c|}
\hline Híbrido & $\begin{array}{c}\text { Rendimiento } \\
(\mathrm{kg} / \mathrm{ha})\end{array}$ & $\begin{array}{l}\text { Floración } \\
\text { (días) }\end{array}$ & $\begin{array}{l}\text { Altura de planta } \\
(\mathrm{cm})\end{array}$ & $\begin{array}{c}\text { Altura de } \\
\text { mazorca }(\mathbf{c m})\end{array}$ & Sanidad \\
\hline UHT96E410 & 12.421 & 77,0 & 209 & 114 & 9,0 \\
\hline UHT96E415 & 11.638 & 84,7 & 235 & 145 & 9,6 \\
\hline UHT96E416 & 11.333 & 88,7 & 229 & 149 & 9,0 \\
\hline UHT96E418 & 10.588 & 80,3 & 227 & 150 & 8,3 \\
\hline UHT96E417 & 9.845 & 83,7 & 232 & 157 & 8,0 \\
\hline UHT96E408 & 9.824 & 79,0 & 208 & 120 & 8,3 \\
\hline UHT96E409 & 9.587 & 77,3 & 207 & 116 & 9,0 \\
\hline UHT96E412 & 9.048 & 80,7 & 216 & 132 & 7,6 \\
\hline UHT96E407 & 8.749 & 79,3 & 216 & 134 & 8,6 \\
\hline A-791 & 8.649 & 93,7 & 244 & 151 & 9,0 \\
\hline UHT96E420 & 8.444 & 79,7 & 198 & 125 & 8,3 \\
\hline UHT96E406 & 8.301 & 82,3 & 213 & 125 & 8,3 \\
\hline UHT96E414 & 8.275 & 81,3 & 204 & 121 & 8,6 \\
\hline UHT96E413 & 8.177 & 78,3 & 211 & 126 & 8,3 \\
\hline P-1157 & 8.019 & 84,7 & 231 & 155 & 8,3 \\
\hline H-137 & 7.984 & 82,7 & 248 & 172 & 8,0 \\
\hline P-1075T & 7.781 & 78,7 & 264 & 160 & 7,6 \\
\hline UHD96E401 & 7.419 & 87,3 & 210 & 137 & 7,0 \\
\hline UHD96E403 & 6.963 & 87,3 & 200 & 126 & 7,6 \\
\hline $\mathrm{H}-135$ & 6.903 & 89,3 & 247 & 164 & 8,6 \\
\hline UHD96E402 & 6.801 & 88,7 & 225 & 143 & 7,0 \\
\hline UHD96E404 & 6.538 & 94,0 & 210 & 145 & 7,6 \\
\hline UHT96E411 & 6.489 & 76,0 & 182 & 111 & 7,6 \\
\hline UHT96E405 & 6.263 & 81,3 & 196 & 121 & 8,0 \\
\hline P-1159 & 6.193 & 81,7 & 212 & 135 & 7,3 \\
\hline UHT96E419 & 5.035 & 78,0 & 213 & 121 & 8,6 \\
\hline D.M.S. & $3.955,8$ & 6,64 & 42,03 & 29,14 & 1,76 \\
\hline
\end{tabular}

incluyendo a los testigos. Queda confirmado que las combinaciones trilineales son una buena opción (Espinosa et al, 1995), ya que además de su productividad de grano, presenta ventajas en la producción de semilla, al ser menos complicada su conformación y más sencillo mantener su calidad genética (Espinosa et al, 1995). Otras de las buenas características que se observan para esta nuevas cruzas triples son su precocidad, ya que florearon desde 77 a 88 días contra 89 y 94 días de H135 y el A-791 respectivamente, así como su altura de planta que va de 207 a 235 centímetros, y la de mazorca de 114 a 157 centímetros, que les da una mayor resistencia al acame (Cruz et al, 1995), así como su excelente sanidad de mazorca, todo esto los hace atractivos para los productores de la zona, aunado a sus excelentes rendimientos (Espinosa et al, 1995b). 


\section{CONCLUSIONES}

Los nuevos híbridos PUMAS de conformación trilineal UHT96E410, UHT96E415, UHT96E416 y UHT96E418 superaron numérica y/o estadísticamente a los testigos comerciales tanto trilineales como de cruza doble, en cuanto a capacidad de rendimiento.

A su vez, éstos nuevos híbridos presentaron mayor precocidad, menor altura de planta y mazorca, y mayor sanidad con respecto a los testigos lo cual les da ventaja en relación a tolerancia al acame, así como el permitir su cosecha mecánica y el mejor manejo agronómico.

Si estos nuevos híbridos siguen mostrando estas bondades, y sobre todo su capacidad productiva, en posteriores evaluaciones, podrán ser una buena alternativa para los productores de la zona de transición entre el Bajío - Valles Altos de la República Mexicana, ya que además dada su conformación trilineal es más rendidora en la producción de grano y presenta ventajas para la producción de semilla.

\section{LITERATURA CITADA}

CRUZ, G.P.; ESPINOSA, C.A.; TADEO R. 1995. Capacidad productiva de híbridos de maíz bajo dos densidades de población en el Valle del Mezquital. In: Investigación multidisciplinaria. Memorias del IX Foro Interno de Investigación. FES-C UNAM, P. 38-41. Cuautitlán, Izcalli, México.

ESPINOSA, C. A.; TADEO, R.M.; MARTÍNEZ,R. M. 1995a. Generación de híbridos de maíz de Valles Altos de México y su empleo en microempresas de semillas. Agronomía Mesoamericana (C.R.) Vol. 6: 148-150.

ESPINOSA, C. A.; TADEO, R.M.; PIÑA,A. D. 1995b. Estabilidad de rendimiento en híbridos de maíz por diferente orden de cruza en la producción de semilla. In: Agronomía Mesoamericana (C.R.). Vol. 6: 98-103.

TADEO, R. M. 1994. Puma 1157 y Puma 1159 maíces híbridos de la UNAM para el campo mexicano. In: Investigación Multidisciplinaria. Coordinación General de Investigación. FES-C UNAM, Cuautitlán Izcalli, México. pp. 25-27.

TADEO, R. M.; ESPINOSA, C.A.; CRUZ,P. G.; PIÑA,A. D.; MARTÍNEZ, M. A. 1996. Rendimiento de los híbridos de maíz PUMA1157 y PUMA 1159 en Tlaxcoapan, Hidalgo. In: Investigación Multidisciplinaria. UNAM, Campus Cuautitlán, Cuautitlán Izcalli, México. pp. 23-26. 